\title{
A Cross-Sectional Study of Retired Great British Olympians (Berlin 1936-Sochi 2014): Olympic Career Injuries, Joint Health in Later Life, and Reasons for Retirement from Olympic Sport
}

\author{
Dale J. Cooper ${ }^{{ }^{*}}$ D, Mark E. Batt ${ }^{2}$, Mary S. O'Hanlon ${ }^{3}$ and Debbie Palmer ${ }^{4}$
}

\begin{abstract}
Background: The relationship between Olympic career sport injury and the long-term musculoskeletal health of the elite athlete remains unclear. This study describes the lifetime prevalence of medical attention injuries that occurred during training and/or competition as part of the athlete's Olympic career, reasons for retirement from Olympic sport, and the point prevalence of pain and osteoarthritis (OA) among retired Great Britain's (GB) Olympians.
\end{abstract}

Methods: This cross-sectional study involved distributing a questionnaire to retired GB Olympians who had competed at 36 Olympic Games between Berlin 1936 and Sochi 2014. The questionnaire captured Olympic career injury history (lasting $\geq 1$ month), sport exposure, musculoskeletal pain (last 4 weeks), physician-diagnosed OA, and joint replacement. Injury prevalence was calculated for sports with a minimal of 15 respondents. Adjusted odds ratios (aOR) were estimated in logistic regression for pain, OA, and joint replacement. Models were adjusted for age, sex, BMl, and career duration.

Results: Six hundred fifty (57.8\% male; $42.2 \%$ female) retired athletes representing 40 sports (29 summer; 11 winter), aged 60.5 years (range 23-97), completed the questionnaire. Overall, 721 injuries (368 athletes) were self-reported equating to a lifetime Olympic career injury prevalence of $56.6 \%$. Injury prevalence was highest in field athletics (81.0\%), gymnastics (75.0\%), and track athletics (67.7\%). Injuries most frequently occurred at the knee (19.0\%), lower back (15.4\%), and shoulder (11.5\%). Of those injured, 19.5\% retired from sport due to injury. Pain was most prevalent at the lumbar spine (32.8\%), knee (25.3\%), and hip (22.5\%), and OA at the knee (13.4\%), hip (10.4\%), and lumbar spine (4.6\%). Injury was associated with pain at the hip (aOR 4.88; 95\% Cl, 1.87-12.72, $\mathrm{p}=0.001$ ), knee (aOR 2.35; $95 \% \mathrm{Cl}, 1.45-3.81, \mathrm{p}=0.001)$, and lumbar spine ( $\mathrm{aOR} 2.53 ; 95 \% \mathrm{Cl}, 1.63-3.92, \mathrm{p}<0.001)$; OA at the hip (aOR 5.97; $95 \% \mathrm{Cl}, 1.59-22.47, \mathrm{p}=0.008$ ) and knee (aOR 3.91; 95\% Cl, 2.21-6.94, $\mathrm{p}<0.001$ ); and joint replacement at the hip (aOR 8.71; 95\% Cl, 2.13-35.63, $\mathrm{p}=0.003$ ) and knee (aOR 5.29; 95\% Cl, 2.39-11.74, $\mathrm{p}<0.001)$.

Conclusion: The lifetime prevalence of Olympic career injury was $56.6 \%$, with those injured more likely to selfreport current pain and/or OA at the hip, knee, and lumbar spine and joint replacement at the hip and knee.

Keywords: Prevalence, Injury, Pain, Osteoarthritis, Olympians

\footnotetext{
* Correspondence: Dalejcooper@hotmail.com

${ }^{1}$ School of Allied Health Professions, Keele University, Keele, Staffordshire, UK Full list of author information is available at the end of the article
}

\section{Springer Open}

(- The Author(s). 2021 Open Access This article is licensed under a Creative Commons Attribution 4.0 International License, which permits use, sharing, adaptation, distribution and reproduction in any medium or format, as long as you give appropriate credit to the original author(s) and the source, provide a link to the Creative Commons licence, and indicate if changes were made. The images or other third party material in this article are included in the article's Creative Commons licence, unless indicated otherwise in a credit line to the material. If material is not included in the article's Creative Commons licence and your intended use is not permitted by statutory regulation or exceeds the permitted use, you will need to obtain permission directly from the copyright holder. To view a copy of this licence, visit http://creativecommons.org/licenses/by/4.0/. 


\section{Key Points}

- Medical attention injuries (lasting $\geq 1$ month) were sustained by $56.6 \%$; injuries occurred most frequently at the knee, lumbar spine, and shoulder with $19.5 \%$ of those injured forced to retire early.

- Fractures, dislocations, and head injuries occurred more frequently in winter sports; females were more at risk of stress fractures, and injuries were associated with pain and osteoarthritis in later life.

- Prevention strategies should aim to reduce injuries in the primary weight-bearing joints of the lower limbs to mitigate the risk of early onset of osteoarthritis and joint replacement in retirement.

\section{Background}

A key mandate of the International Olympic Committee (IOC) is to encourage and support measures that protect the health of the athlete in sport $[1,2]$. The IOC has promoted epidemiological studies for identifying and reducing injuries and maximising athlete health [1]. The monitoring of injuries was implemented for the first time at the 2008 Beijing Olympic Games [3], and the surveillance of both injuries and illnesses was subsequently introduced at the 2010 Vancouver Olympic Games [4]. Published literature from injury surveillance studies have provided a description of the types and mechanisms of injuries sustained in current elite athletes on a seasonal basis and during major sporting events [5-10]. However, the relationship between sport injury and the long-term musculoskeletal health of the elite athlete remains unclear.

Previous studies have indicated, compared with population controls, that former elite athletes have a lower risk of stroke, cancer, diabetes, heart disease, and mortality in later life [11-13]. Whereas the degree of musculoskeletal morbidity has been shown to be higher in former male athletes from Olympic sports [14], soccer [15], cricket [12], and Rugby Union [13]. Musculoskeletal injuries are common in elite athletes and these can lead to early post-traumatic osteoarthritis (PTOA) [16, 17]. Post-traumatic OA progresses more rapidly to endstage disease compared to idiopathic OA and can impair health-related quality of life $[16,18]$. To date, retiredathlete studies have investigated and reported an association between injury with OA but these studies have focused on male athletes and at isolated joints $[14,15,19]$.

To our knowledge, no epidemiological studies have accounted for the occurrence of lifetime career injuries and the prevalence of pain and OA across multiple body sites in a retired Olympic male and female athletic population. If we are to prevent injury, premature retirement, and the sequelae of joint disease in retired elite athletes, knowledge is required of the nature of lifetime and career-ending injuries, the joints most commonly affected by joint disease, and the nature of injuries that occurred at those joints. The aims of this study are to describe the lifetime prevalence of medical attention injuries that occurred during training and/or competition as part of the athlete's Olympic career, the reasons for retirement from Olympic sport, and the point prevalence of pain and OA among retired Great Britain's (GB) Olympians.

\section{Methods \\ Study Design}

This cross-sectional study involved distributing a letter by post or email inviting GB Olympians the opportunity to complete and return a postal questionnaire or the option of completing an online version. The questionnaire was distributed to both current and retired athletes registered on the British Olympic Association (BOA) database. For the present study, we excluded responses from those who were training to qualify for or compete at any upcoming Olympic Games. The first phase of data collection took place between May 2014 and April 2015 and involved distributing the questionnaire to those living in the UK and to those residing overseas. Two email reminders and one postal reminder were sent to nonresponders. The second phase of data collection involved the BOA Athlete's Commission distributing individual copies of the questionnaire. All questionnaires were returned by December 2016.

\section{Data Collection and Management}

The study questionnaire content is reported elsewhere [20], and data collected on demographics, sports career participation, medical history, musculoskeletal health, and Olympic career injury are presented in this study. Baseline questions captured self-reported information on age (years), sex, height $(\mathrm{cm})$, weight $(\mathrm{kg})$, and ethnicity. Early life (i.e. during the 20s) and current height and weight data were self-reported and used to calculate Olympic career and current body mass index (BMI kg/ $\mathrm{m}^{2}$ ) separately. Sport participation included the frequency and duration for the period of training and competition leading up to their first Olympic Games until retirement from their last Olympic Games. The presence of a significant injury was determined by asking participants: "have you ever sustained a significant injury that caused pain for most days during a 1-month period or more and for which you consulted a medical professional or a health provider such as a general practitioner?" [20]. Injury data was collected in line with IOC injury surveillance methods and included anatomical location, type, mechanism of injury, competition, and training injuries $[8,9,21,22]$. Participants were asked to self-report the location and duration of current pain 
within the last month [23]. A body manikin was used to self-report the location of pain using a method shown to be repeatable [24]. Injuries and bodily pain lasting less than 1 month were not recorded and non-sport injuries were excluded. The presence of OA was determined by asking participants: "have you ever been diagnosed with $\mathrm{OA}$ in any of your joints by a physician, and if so, please state which joint/s?" Participants were asked to record the date, type, and reasons for joint replacement surgery. Where GB Olympians had competed in at least two disciplines at Olympic level, preference was given to the discipline in which the participant had spent the longest time competing.

\section{Definitions}

A consensus meeting took place with 14 retired GB Olympic athletes at the BOA to establish agreement for defining Olympic career injury and retirement from Olympic sport. A retired Olympian was defined as 'an Olympian who was retired from Olympic competition (i.e. those who had confirmed they had retired and were no longer training to qualify for or compete at any upcoming Olympic Games)'. A significant Olympic career injury was adapted from the IOC approach for recording injury in multi-sport events [22]. Injury was defined as 'an injury that required medical attention irrespective of absence from competition or training and occurred during training for, and/or competition, as part of the athlete's Olympic career and caused pain for most days during a 1-month period or more'. In agreement with consensus statements, recurrent injury was defined by the same location and type, which occurred after an athlete returned to full participation from the previous injury $[22,25]$.

\section{Statistical Analysis}

Statistical analyses were conducted within SPSS 25.0 (SPSS Inc., Chicago, IL, USA). Descriptive statistics are presented as frequencies (proportion) for categorical variables, and for numerical variables data are presented with the mean and standard deviation, or median and range where data are not normally distributed. The prevalence of career injury was calculated by dividing the number of injured athletes by the total number of athletes and presented as percentages (\%) with $95 \%$ confidence intervals (CIs). Crude odds ratios with 95\% CIs were estimated in logistic regression for factors and outcomes of hip and knee replacement. These models were adjusted with staged adjustment for the potential confounders of age, sex, BMI, injury, and Olympic career exposure. The most severe hip and knee joint were selected as the index joint for analysis. Significant injuries were matched according to the index joint and included if they preceded the date of OA diagnosis and joint replacement. Significance was accepted at p $<0.05$ and imputation was not undertaken for missing values.

\section{Power Calculation}

The power calculation was performed using GPower V.3.1.9.2. In a sample of 650, with a 1:1 ratio for exposed and unexposed, we had at least $80 \%$ power to detect an odds ratio of 1.96 and 2.16 or greater at $5 \%$ significance based on a prevalence rate of $7 \%$ and $5 \%$ for hip and knee replacement one-tailed (assuming those with a joint injury would have greater risk), respectively.

\section{Research Ethics Approval}

This study was approved by the University of Nottingham Research Ethics Committee (Reference No: K13022014). All procedures involving research participants were in accordance with the ethical standards of the university institution review board and with the 1964 Helsinki Declaration and its later amendments. All participants received written information at the start of the study, detailing how data would be stored and treated confidentially, ensuring athlete anonymity at all times. It was made explicitly clear to participants that by completing and returning a questionnaire they gave implied consent for their data to be used anonymously for the purposes of this study.

\section{Results \\ Olympian Characteristics}

A total of 2742 questionnaires were distributed to current and retired athletes and 743 returned a completed questionnaire (27.1\% response rate). Of the 743 returns achieved, 650 were retired from their Olympic career and had data for the prevalence analysis of injury (Fig. 1). Table 1 reports the characteristics of the respondents (male 57.8\%; female 42.2\%) that were aged between 23 and 97 years (median 60.5 years). Respondents had competed (mean $9.8 \pm 6.2$ years) in at least one of 40 Olympic sports (Table 2). Of the 650 respondents, 66 had competed in 11 sports at the Winter Olympics and 584 had competed in 29 sports at the Summer Olympics. All participants were retired (mean $31.2 \pm 16.7$ years) from their Olympic careers and participation spanned Summer and Winter Olympic Games from Berlin in 1936 to Sochi in 2014.

\section{Prevalence of Injury}

There were 721 injuries (368 athletes) reported from 650 retired GB Olympians equating to $56.6 \%$ (95\% CI $52.8-$ 60.4) of Olympians reporting at least one significant Olympic career-related injury. Each Olympian reported a mean of 1.11 injuries (female $=1.35$; male $=0.94$ ) during their Olympic career, with $50.8 \%(\mathrm{n}=366)$ and $37.7 \%(\mathrm{n}=272)$ of injuries attributed to training and competition, respectively (Table 2). Injury prevalence 


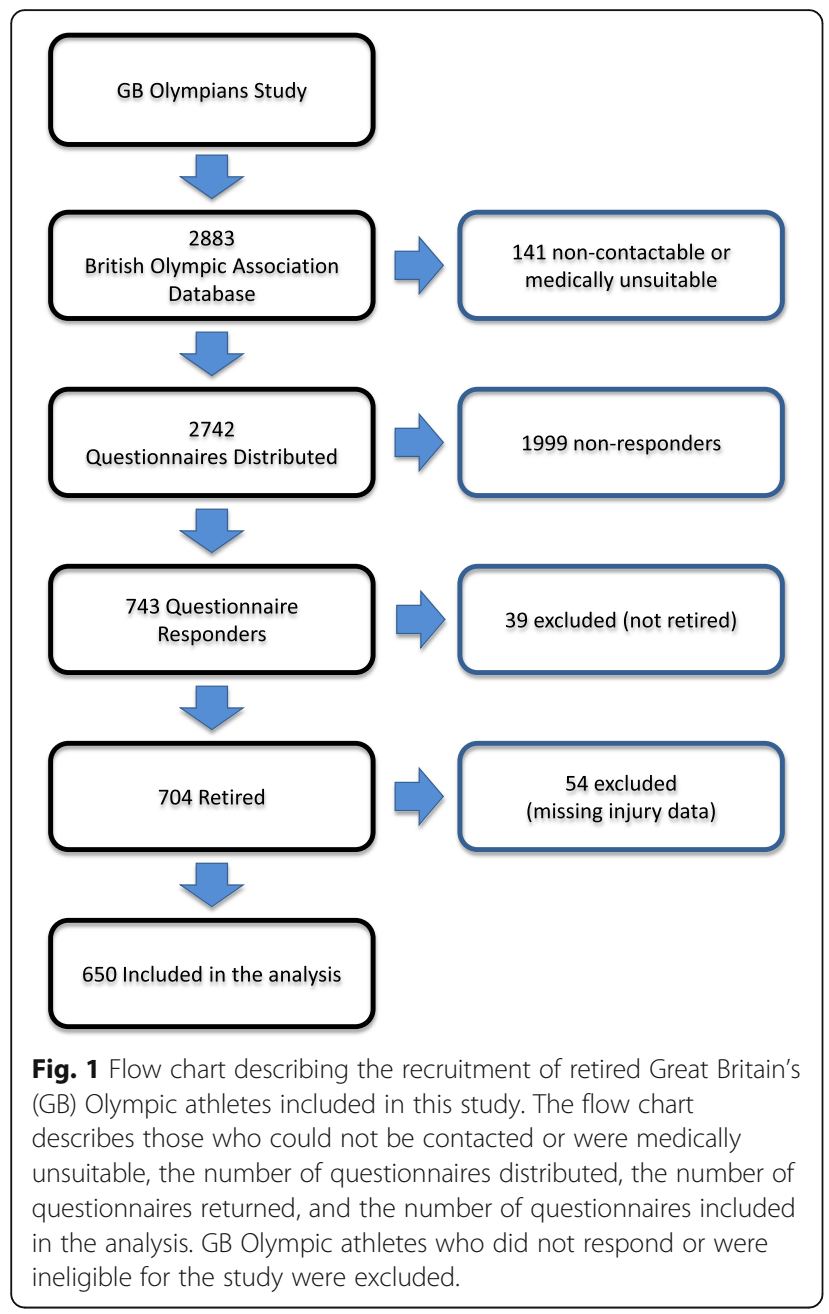

Fig. 1 Flow chart describing the recruitment of retired Great Britain's (GB) Olympic athletes included in this study. The flow chart describes those who could not be contacted or were medically unsuitable, the number of questionnaires distributed, the number of in the analysis. GB Olympic athletes who did not respond or were ineligible for the study were excluded. was $48.5 \%$ (36.1-60.9) for winter Olympians compared with 57.5\% (53.5-61.6) for summer Olympians. After adjustment (age, sex, BMI, and career duration), there was no significant difference in the number of injured athletes competing at the Winter Olympics compared to the Summer Olympics (aOR 1.00; 95\% CI, 0.98-1.02, p $=0.956$ ). However, participants from weight-bearing sports were more likely to self-report a significant injury (aOR 1.69; 95\% CI, 1.22, 2.36, $\mathrm{p}=.002$ ). By sport, injury prevalence was highest in field athletics $(81.0 \%$ (62.699.3)), gymnastics $(75.0 \%(54.2-95.8))$, track athletics $(67.7 \%$ (58.3-77.1)), and lowest for canoeing (28.6\% (10.7-46.4)), swimming (46.6\% (34.9-58.3)), and sailing (50.0\% (26.0-74.0)) (sports with $\mathrm{n} \geq 15$ participants).

\section{Injury Location, Type, and Cause}

Injuries most frequently occurred at the knee $(19.0 \%$ (137/721)), lower back (15.4\% (111/721)), and shoulder (11.5\% (83/721)) (Table 3). The most common injury types were ligament sprain $(19.3 \%(139 / 721))$, traumatic fractures $(18.4 \%(133 / 721))$, and muscle injury (12.2\% (88/721)) (Table 4). Traumatic fractures accounted for $22.2 \%(78 / 352)$ of all injuries in males and $14.9 \%$ (55/ 369) in females; stress fractures accounted for $3.1 \%$ (11/ $352)$ of all injuries in males and $6.8 \%(25 / 369)$ in females. Females were no more likely to report any injury (aOR $0.84 ; 95 \% \mathrm{CI}, 0.59-1.18, \mathrm{p}=0.32$ ) or traumatic fracture than males $(\mathrm{aOR} 1.33$; 95\% CI, 0.82-2.16, $\mathrm{p}=$ 0.243 ); however, females were more likely to report a stress fracture (aOR 2.85; 95\% CI, 1.15-7.04, $\mathrm{p}=0.023$ ). There was a single catastrophic injury with spinal cord injury and paraplegia as the outcome.

The most prominent injury locations in GB Olympians who had participated in the Summer Olympics were the knee (18.6\% (122/655)), lower back (15.7\% (103/655)), and shoulder $(10.8 \%(71 / 655))$, and this compares to the

Table 1 Olympian characteristics

\begin{tabular}{|c|c|c|c|c|}
\hline & All, $n=650$ & Female, $n=274$ & Male, $\mathrm{n}=376$ & $P$ values \\
\hline \multicolumn{5}{|l|}{ Anthropometrics } \\
\hline Age (years), median (range) & $60.5(23-97)$ & $54(23-93)$ & $66(26-97)$ & $\mathrm{p}<0.001$ \\
\hline Height (cm), mean (SD) & $175.6(10.2)$ & $169.0(7.6)$ & $180.4(9.0)$ & $\mathrm{p}<0.001$ \\
\hline Weight (kg), mean (SD) & $76.6(15.8)$ & $67.8(13.8)$ & $83.1(14.0)$ & $\mathrm{p}<0.001$ \\
\hline Current BMI $\left(\mathrm{kg} / \mathrm{m}^{2}\right)$, mean (SD) & $24.7(4.0)$ & $23.7(4.7)$ & $25.4(3.3)$ & $\mathrm{p}<0.001$ \\
\hline Olympic career BMI $\left(\mathrm{kg} / \mathrm{m}^{2}\right)$, mean (SD) & $22.8(2.9)$ & $21.7(2.5)$ & $23.5(2.9)$ & $\mathrm{p}<0.001$ \\
\hline \multicolumn{5}{|l|}{ Sport exposure } \\
\hline Athletes by summer/winter sport, n (\%) & $\begin{array}{l}274(42.2) \\
376(57.8)\end{array}$ & $\begin{array}{l}250(91.2) \\
24(8.8)\end{array}$ & $\begin{array}{l}334(88.8) \\
42(11.2)\end{array}$ & $p=0.382$ \\
\hline Number of international years doing Olympic sport, mean (SD) & $9.8(6.2)$ & $10.2(6.8)$ & $9.4(5.7)$ & $p=0.301$ \\
\hline Number of years retired from Olympic participation, mean (SD) & $31.2(16.7)$ & $27.5(16.2)$ & $34.2(16.5)$ & $\mathrm{p}<0.001$ \\
\hline
\end{tabular}

A comparison for differences between the groups was analysed using the unpaired $t$ test for continuous variables, or Mann-Whitney $\mathrm{U}$ test where appropriate, and categorical variables were analysed using $x^{2}$. Statistically significant differences are highlighted in bold 
Table 2 Injuries by Olympic sport and sex in retired Great Britain's Olympians

\begin{tabular}{|c|c|c|c|c|c|c|c|}
\hline & $\begin{array}{l}\text { Athletes } \\
(\mathrm{n} ; \mathrm{m} / \mathrm{f})^{*}\end{array}$ & Injuries (n) & $\begin{array}{l}\text { Training/competition/ } \\
\text { unknown** (\%) }\end{array}$ & $\begin{array}{l}\text { Injuries per } \\
\text { athlete } \\
\text { (mean } n \text { ) }\end{array}$ & $\begin{array}{l}\text { Injured athletes } \\
(n ; m / f)\end{array}$ & $\begin{array}{l}\text { Injury prevalence } \\
(95 \% \mathrm{Cl})\end{array}$ & Exact $95 \% \mathrm{Cl}^{* * * *}$ \\
\hline \multicolumn{8}{|l|}{ Summer sports } \\
\hline Archery & $10(3 / 7)$ & 3 & 0/66.7/33.3 & 0.30 & $3(1 / 2)$ & - & \\
\hline Athletics (track) & $99(53 / 46)$ & 128 & 62.5/28.9/8.6 & 1.29 & $67(35 / 32)$ & $67.7 \%$ & 58.3 to 77.1 \\
\hline Athletics (field) & $21(8 / 13)$ & 29 & 37.9/48.3/13.8 & 1.38 & $17(7 / 10)$ & $81.0 \%$ & 62.6 to 99.3 \\
\hline Athletics (road) & $19(12 / 7)$ & 30 & $53.3 / 43.3 / 3.4$ & 1.58 & $10(7 / 3)$ & $52.6 \%$ & 27.9 to 77.4 \\
\hline \multicolumn{8}{|l|}{ Aquatics } \\
\hline Swimming & $73(26 / 47)$ & 58 & $50 / 25.9 / 24.1$ & 0.79 & $34(8 / 26)$ & $46.6 \%$ & 34.9 to 58.3 \\
\hline Diving & $13(4 / 9)$ & 17 & $58.8 / 23.5 / 17.7$ & 1.31 & $8(1 / 7)$ & - & \\
\hline Syn. swimming & $3(0 / 3)$ & 5 & 100/0/0 & 1.67 & $2(0 / 2)$ & - & \\
\hline Water polo & $4(3 / 1)$ & 6 & 100/0/0 & 1.50 & $2(1 / 1)$ & - & \\
\hline Badminton & $4(2 / 2)$ & 4 & $75 / 0 / 25$ & 1.00 & $2(2 / 0)$ & - & \\
\hline Basketball & $4(3 / 1)$ & 6 & $33.3 / 50 / 16.7$ & 1.50 & $3(2 / 1)$ & - & \\
\hline Boxing & $6(6 / 0)$ & 1 & 0/0/100 & 0.17 & $1(1 / 0)$ & - & \\
\hline Canoeing & $28(17 / 11)$ & 13 & $76.9 / 7.7 / 15.4$ & 0.46 & $8(3 / 5)$ & $28.6 \%$ & 10.7 to 46.4 \\
\hline Cycling & $23(17 / 6)$ & 28 & 25/67.9/7.1 & 1.22 & $15(10 / 5)$ & $65.2 \%$ & 44.2 to 86.3 \\
\hline Equestrian & $10(3 / 7)$ & 15 & $26.7 / 60 / 13.3$ & 1.50 & $5(2 / 3)$ & - & \\
\hline Fencing & $23(13 / 10)$ & 18 & 33.3/55.6/11.1 & 0.78 & $12(6 / 6)$ & $52.2 \%$ & 30.1 to 74.3 \\
\hline Football & $5(5 / 0)$ & 5 & 0/80/20 & 1.00 & $4(4 / 0)$ & - & \\
\hline Gymnastics & $20(9 / 11)$ & 31 & $64.5 / 32.3 / 3.2$ & 1.55 & $15(7 / 8)$ & $75.0 \%$ & 54.2 to 95.8 \\
\hline Handball & $1(1 / 0)$ & 0 & - & 0.00 & - & - & \\
\hline Hockey & $57(46 / 11)$ & 62 & 25.8/61.3/12.9 & 1.09 & $33(26 / 7)$ & $57.9 \%$ & 44.7 to 71.1 \\
\hline Judo & $10(6 / 4)$ & 26 & $46.2 / 50 / 3.8$ & 2.60 & $7(4 / 3)$ & - & \\
\hline Modern pentathlon & $7(5 / 2)$ & 11 & $54.5 / 36.4 / 9.1$ & 1.57 & $6(4 / 2)$ & - & \\
\hline Rowing & $93(54 / 39)$ & 93 & $67.7 / 21.5 / 10.8$ & 1.00 & $50(26 / 24)$ & $53.8 \%$ & 43.4 to 64.1 \\
\hline Sailing & $20(15 / 5)$ & 20 & $45 / 25 / 30$ & 1.00 & $10(5 / 5)$ & $50.0 \%$ & 26.0 to 74.0 \\
\hline Shooting & $10(6 / 4)$ & 10 & $30 / 70 / 0$ & 1.00 & $7(4 / 3)$ & - & \\
\hline Table tennis & $1(1 / 0)$ & 2 & 0/100/0 & 2.00 & $1(1 / 0)$ & - & \\
\hline Taekwondo & $1(0 / 1)$ & 0 & - & 0.00 & - & - & \\
\hline Tennis & $5(4 / 1)$ & 7 & $14.3 / 85.7 / 0$ & 1.40 & $2(1 / 1)$ & - & \\
\hline Volleyball & $2(2 / 0)$ & 3 & $100 / 0 / 0$ & 1.50 & $1(1 / 0)$ & - & \\
\hline Weightlifting & $8(6 / 2)$ & 19 & 47.4/42.1/10.5 & 2.38 & $7(5 / 2)$ & - & \\
\hline Wind surfing & $1(1 / 0)$ & 1 & 0/0/100 & 1.00 & $1(1 / 0)$ & - & \\
\hline Wrestling & $3(3 / 0)$ & 4 & $25 / 50 / 25$ & 1.33 & $3(3 / 0)$ & - & \\
\hline Winter sports & & & & & & - & \\
\hline Alpine skiing & $12(7 / 5)$ & 14 & $6 / 5 / 3$ & 1.17 & $2(2 / 0)$ & - & \\
\hline Biathlon & $4(4 / 0)$ & 4 & $50 / 50 / 0$ & 1.00 & $2(2 / 0)$ & - & \\
\hline Bobsleigh & $15(13 / 2)$ & 13 & $53.8 / 23.1 / 23.1$ & 0.87 & $9(7 / 2)$ & $60.0 \%$ & 31.9 to 88.1 \\
\hline Cross-country skiing & $9(6 / 3)$ & 11 & $18.2 / 81.8 / 0$ & 1.22 & $3(3 / 0)$ & - & \\
\hline Figure skating & $10(2 / 8)$ & 5 & 100/0/0 & 0.50 & $5(1 / 4)$ & - & \\
\hline Ice hockey & $1(1 / 0)$ & 1 & 0/100/0 & 1.00 & $1(1 / 0)$ & - & \\
\hline Luge & $3(3 / 0)$ & 3 & $33.3 / 66.7 / 0$ & 1.00 & $2(2 / 0)$ & - & \\
\hline Short tr. sp. skating & $7(3 / 4)$ & 7 & $85.7 / 14.3 / 0$ & 1.00 & $5(2 / 3)$ & - & \\
\hline Skeleton & $1(0 / 1)$ & 1 & 100/0/0 & 1.00 & $1(0 / 1)$ & - & \\
\hline
\end{tabular}


Table 2 Injuries by Olympic sport and sex in retired Great Britain's Olympians (Continued)

\begin{tabular}{|c|c|c|c|c|c|c|c|}
\hline & $\begin{array}{l}\text { Athletes } \\
(\mathrm{n} ; \mathrm{m} / \mathrm{f})^{*}\end{array}$ & Injuries (n) & $\begin{array}{l}\text { Training/competition/ } \\
\text { unknown** (\%) }\end{array}$ & $\begin{array}{l}\text { Injuries per } \\
\text { athlete } \\
\text { (mean } n \text { ) }\end{array}$ & $\begin{array}{l}\text { Injured athletes } \\
(\mathrm{n} ; \mathrm{m} / \mathrm{f})\end{array}$ & $\begin{array}{l}\text { Injury prevalence } \\
(95 \% \mathrm{Cl})\end{array}$ & Exact $95 \% \mathrm{Cl}^{* * *}$ \\
\hline Skiing: freestyle & $2(1 / 1)$ & 7 & $57.1 / 42.9 / 0$ & 3.50 & $2(1 / 1)$ & - & \\
\hline Speed skating & $2(2 / 0)$ & 0 & - & - & - & - & \\
\hline Total summer sports & $584(334 / 250)$ & 655 & $50.7 / 37.6 / 11.7$ & 1.12 & $336(178 / 158)$ & $57.5 \%$ & 53.5 to 61.6 \\
\hline Total winter sports & $66(42 / 24)$ & 66 & 51.5/39.4/9.1 & 1.00 & $32(21 / 11)$ & $48.5 \%$ & 36.1 to 60.9 \\
\hline Overall total & 650 & 721 & $50.8 / 37.7 / 11.5$ & 1.11 & $368(199 / 169)$ & $56.6 \%$ & 52.8 to 60.4 \\
\hline
\end{tabular}

${ }^{*} m$ males, $f$ females

**Occurred in training, competition, unknown (i.e. competition or training)

${ }^{* * *} \mathrm{Cl}$ confidence intervals; prevalence not reported for sports with $<15$ Olympians

knee $(22.7 \%(15 / 66))$, shoulder $(18.2 \%(12 / 66))$, and lower back (12.1\% (8/66)) among those who had participated in the Winter Olympics. The number of sportrelated injuries in the Summer and Winter Olympics were comparable: competition $37.6 \%(246 / 655)$ versus $39.4 \%(26 / 66)$ and training $50.7 \%(332 / 655)$ versus $51.5 \%$ (34/66), respectively (Table 2 ). The most common reported injury mechanisms were non-contact trauma $40.3 \%$ (85/211), overuse gradual onset 30.3\% (64/211), and sudden onset $16.6 \%(35 / 211)$.

\section{Reasons for Retirement from Olympic Career}

Of the participants included in this study, 19.5\% (127/ 650) reported they had retired early from their Olympic careers because of injury. Of those forced to retire due to injury, $32.3 \%(41 / 127)$ had suffered a recurrent injury and $26.0 \%(33 / 127)$ reported retiring due to a one-off injury. The main locations of injuries responsible for retirement from sport were the lumbar spine $(26.0 \%$ (33/ 127)), knee (24.4\% (31/127)), ankle (14.2\% (18/127)), lower leg $(8.7 \%(11 / 127))$, shoulder $(7.1 \%(9 / 127))$, and

Table 3 Location of injuries (\%) in retired Great Britain's Olympians

\begin{tabular}{|c|c|c|c|}
\hline Body part & Number of female injuries (\%) & Number of male injuries (\%) & Total number of injuries (\%) \\
\hline Knee & $69(18.7)$ & $68(19.3)$ & $137(19.0)$ \\
\hline Lower back & $61(16.5)$ & $50(14.2)$ & $111(15.4)$ \\
\hline Shoulder & $33(8.9)$ & $50(14.2)$ & $83(11.5)$ \\
\hline Ankle & $38(10.3)$ & $29(8.2)$ & $67(9.3)$ \\
\hline Others/unknown & $28(7.6)$ & $27(7.7)$ & $55(7.6)$ \\
\hline Lower leg & $21(5.7)$ & $27(7.7)$ & $48(6.7)$ \\
\hline Foot/toe & $21(5.7)$ & $11(3.1)$ & $32(4.4)$ \\
\hline Thigh & $11(3.0)$ & $15(4.3)$ & $26(3.6)$ \\
\hline Achilles ten. & $13(3.5)$ & $13(3.7)$ & $26(3.6)$ \\
\hline Wrist & $14(3.8)$ & $11(3.1)$ & $25(3.5)$ \\
\hline Elbow & $16(4.3)$ & $4(1.1)$ & $20(2.8)$ \\
\hline Head & $6(1.6)$ & $10(2.8)$ & $16(2.2)$ \\
\hline Hip & $6(1.6)$ & $7(2.0)$ & $13(1.8)$ \\
\hline Sternum/rib & $8(2.2)$ & $5(1.4)$ & $13(1.8)$ \\
\hline Neck & $5(1.4)$ & $5(1.4)$ & $10(1.4)$ \\
\hline Upper back & $6(1.6)$ & $4(1.1)$ & $10(1.4)$ \\
\hline Forearm & $5(1.4)$ & $3(0.9)$ & $8(1.1)$ \\
\hline Finger & $3(0.8)$ & $3(0.9)$ & $6(0.8)$ \\
\hline Hand & $2(0.5)$ & $3(0.9)$ & $5(0.7)$ \\
\hline Pelvis/SIJ & $2(0.5)$ & $1(0.3)$ & $3(0.4)$ \\
\hline Upper arm & - & $3(0.9)$ & $3(0.4)$ \\
\hline Abdomen & - & $2(0.6)$ & $2(0.3)$ \\
\hline Thumb & $1(0.3)$ & $1(0.3)$ & $2(0.3)$ \\
\hline Total & 369 & 352 & 721 \\
\hline
\end{tabular}


Table 4 Injury type (\%) in retired Great Britain's Olympians

\begin{tabular}{|c|c|c|c|}
\hline Type of injury & Injuries in female (\%) & Injuries in male (\%) & Total number of injuries (\%) \\
\hline Ligament injury (sprain) & $84(22.8)$ & $55(15.6)$ & $139(19.3)$ \\
\hline Fracture (traumatic) & $55(14.9)$ & $78(22.2)$ & $133(18.4)$ \\
\hline Muscle injury (strain) & $44(11.9)$ & $44(12.5)$ & $88(12.2)$ \\
\hline Tendinopathy & $33(8.9)$ & $44(12.5)$ & $77(10.7)$ \\
\hline Cartilage injury & $25(6.8)$ & $27(7.7)$ & $52(7.2)$ \\
\hline Disc prolapse & $24(6.5)$ & $16(4.5)$ & $40(5.5)$ \\
\hline Dislocation/subluxation & $15(4.1)$ & $22(6.3)$ & $37(5.1)$ \\
\hline Stress fracture & $25(6.8)$ & $11(3.1)$ & $36(5.0)$ \\
\hline Nerve injury & $7(1.9)$ & $5(1.4)$ & $12(1.7)$ \\
\hline Contusion/hematoma & $8(2.2)$ & $1(0.3)$ & $9(1.2)$ \\
\hline Compartment syndrome & - & $4(1.1)$ & $4(0.6)$ \\
\hline Fasciitis & $3(0.8)$ & - & $3(0.4)$ \\
\hline Laceration & $2(0.5)$ & $1(0.3)$ & $3(0.4)$ \\
\hline Muscle cramps & $2(0.5)$ & $1(0.3)$ & $3(0.4)$ \\
\hline Concussion & $2(0.5)$ & - & $2(0.3)$ \\
\hline Impingement & - & $2(0.6)$ & $2(0.3)$ \\
\hline Dental injury & - & $1(0.3)$ & $1(0.1)$ \\
\hline Amputation & - & $1(0.3)$ & $1(0.1)$ \\
\hline Medial tibial stress syndrome & $1(0.3)$ & - & $1(0.1)$ \\
\hline Other/missing & 39 (10.6) & $39(11.1)$ & $78(10.8)$ \\
\hline Total & 369 & 352 & 721 \\
\hline
\end{tabular}

hip $(5.5 \%(7 / 127))$. The main types of injury responsible for early retirement from sport were ligament injury (19.7\% (25/127)), intervertebral disc prolapse (18.1\% (23/ $127))$, tendinopathy $(14.2 \% \quad(18 / 127))$ cartilage tears (9.4\% (12/127)), and fracture +/- dislocation (5.5\% (7/ 127)). Approximately $80.5 \%$ (523/650) of all participants retired for non-injury reasons: because they had achieved all that was possible (37.9\% (198/523)), chosen an alternative career $(27.9 \%(146 / 523))$, declined in fitness $(12.6 \%(66 / 523))$, financial reasons, or starting a family $(9.0 \%(47 / 523))$.

\section{Musculoskeletal Health}

The prevalence of pain was highest at the lumbar spine (32.8\% (197/600)), knee (25.3\% (152/600)), and hip $(22.5 \%(135 / 600))$, and OA was most prevalent at the knee (13.4\% (85/635)), hip (10.4\% (66/635)), and lumbar spine $(4.6 \%(29 / 635))$ (Table 5). The prevalence of hip and knee replacement resulting from end-stage OA was 7.0\% (44/628) and 5.4\% (34/628). After adjustment (age, BMI, sex, career duration), injury was associated with pain at the hip (aOR 4.88; 95\% CI, 1.87-12.72, $\mathrm{p}=$ 0.001 ), knee (aOR 2.35; 95\% CI, 1.45-3.81, p = 0.001) and lumbar spine (aOR 2.53; 95\% CI, 1.63-3.92, p < 0.001 ), and OA at the hip (aOR 5.97; 95\% CI, 1.5922.47, $\mathrm{p}=0.008)$ and knee (aOR 3.91; 95\% CI, 2.21-
6.94, $\mathrm{p}<0.001)$. Those injured at the hip, knee, or ankle were more likely to self-report physician-diagnosed OA in these joints before 45 years [aOR 4.28; 95\% CI, 1.08$17.02, \mathrm{p}=0.039$ ]. Injury was the main attributable factor associated with joint replacement at the hip (aOR 8.71; 95\% CI, 2.13-35.63, $\mathrm{p}=0.003$ ) and knee (aOR 5.29; 95\% CI, 2.39-11.74, p < 0.001). A one-unit increase in age was associated with joint replacement at the hip (aOR 1.09, 95\% CI, 1.06-1.12, $\mathrm{p}<0.001$ ) and knee (aOR 1.07; 95\% CI, 1.04-1.10, p < 0.001), with BMI associated with knee replacement (aOR 1.11; 95\% CI, 1.03-1.20, p = 0.004) (Table 6).

\section{Discussion}

The aims of the study were to describe the lifetime prevalence of medical attention injuries that occurred during training and/or competition as part of the athlete's Olympic career, and the point prevalence of pain and OA among retired GB Olympians. The main findings are that (1) $56.6 \%$ of retired Olympians selfreported sustaining at least one significant Olympic career injury; (2) each Olympian reported a mean of 1.11 injuries (females 1.37, males 0.92) during their Olympic career, with the majority of injuries attributed to training; (3) injuries most frequently occurred at the knee, lower back, and shoulder; (4) 19.5\% self-reported they 
Table 5 Musculoskeletal health in retired Great Britain's Olympians

\begin{tabular}{|c|c|c|c|c|c|c|}
\hline & $\begin{array}{l}\text { Women } \\
(n=246)\end{array}$ & $\begin{array}{l}\text { Prevalence \% } \\
(95 \% \text { Cl) }\end{array}$ & $\begin{array}{l}\text { Men } \\
(n=354)\end{array}$ & $\begin{array}{l}\text { Prevalence \% } \\
(95 \% \text { CI) }\end{array}$ & All $(n=600)$ & $\begin{array}{l}\text { Overall prevalence } \\
(95 \% \mathrm{Cl})\end{array}$ \\
\hline \multicolumn{7}{|l|}{ Pain (last 4 weeks) } \\
\hline Lumbar spine & 83 & $33.7(27.8-39.7)$ & 114 & $32.2(27.3-37.1)$ & 197 & $32.8(29.1-36.6)$ \\
\hline Knee & 65 & $26.4(20.9-32.0)$ & 87 & $24.6(20.1-29.1)$ & 152 & $25.3(21.8-28.8)$ \\
\hline Hip & 51 & $20.7(15.6-25.8)$ & 84 & $23.7(19.3-28.2)$ & 135 & $22.5(19.1-25.8)$ \\
\hline Ankle & 38 & $15.4(10.9-20.0)$ & 46 & $13.0(9.5-16.5)$ & 84 & $14.0(11.2-16.8)$ \\
\hline Cervical spine & 21 & $8.5(5.0-12.1)$ & 32 & $9.0(6.0-12.0)$ & 53 & $8.8(6.6-11.1)$ \\
\hline 1st metatarsophalangeal joint & 7 & $2.8(0.8-4.9)$ & 5 & $1.4(1.8-2.6)$ & 12 & $2.0(0.9-3.1)$ \\
\hline \multirow[t]{2}{*}{ 1st carpometacarpal joint } & 6 & $2.4(0.5-4.4)$ & 5 & $1.4(1.8-2.6)$ & 11 & $1.8(0.8-2.9)$ \\
\hline & $\begin{array}{l}\text { Women } \\
(n=268)\end{array}$ & $\begin{array}{l}\text { Prevalence \% } \\
(95 \% \mathrm{Cl})\end{array}$ & $\begin{array}{l}\text { Men } \\
(n=367)\end{array}$ & $\begin{array}{l}\text { Prevalence \% } \\
(95 \% \text { Cl) }\end{array}$ & All $(n=635)$ & $\begin{array}{l}\text { Overall prevalence } \\
(95 \% \mathrm{Cl})\end{array}$ \\
\hline \multicolumn{7}{|c|}{ OA (self-report physician-diagnosed) } \\
\hline Knee & 31 & $11.6(7.7-15.4)$ & 54 & $14.7(11.1-18.4)$ & 85 & $13.4(10.7-16.0)$ \\
\hline Hip & 21 & $7.8(4.6-11.1)$ & 45 & $12.3(8.9-15.6)$ & 66 & $10.4(8.0-12.8)$ \\
\hline Lumbar spine & 20 & $7.5(4.3-10.6) *$ & 9 & $2.5(0.9-4.0)$ & 29 & $4.6(2.9-6.2)$ \\
\hline 1st carpometacarpal joint & 6 & $2.2(0.5-4.0)$ & 8 & $2.2(0.7-3.7)$ & 14 & $2.2(1.1-3.4)$ \\
\hline 1st metatarsophalangeal joint & 5 & $1.9(0.2-3.5)$ & 8 & $2.2(0.7-3.7)$ & 13 & $2.0(0.9-3.2)$ \\
\hline Cervical spine & 5 & $1.9(0.2-3.5)$ & 7 & $1.9(0.5-3.3)$ & 12 & $1.9(0.8-3.0)$ \\
\hline Ankle & 6 & $2.2(0.5-4.0)$ & 5 & $1.4(0.2-2.6)$ & 11 & $1.7(0.7-2.8)$ \\
\hline
\end{tabular}

$\mathrm{p}=<0.01$

*Men to women

retired early from their Olympic careers because of injury; (5) current pain was most prevalent at the lumbar spine (32.8\%), knee (25.3\%), and hip (22.5\%), and OA at the knee (13.4\%), hip (10.4\%), and lumbar spine $(4.6 \%)$; and (6) injury was the main attributable factor associated with pain, OA, and joint replacement at the hip and knee.

There is a paucity of existing data for whole career injuries in Olympians, with only one global study available that was conducted as a follow-up to the current study. Using a similar injury definition, the Retired Olympian Musculoskeletal Health Study (ROMHS) reported Olympic career sports injuries in 3357 former Olympians and found a $63.0 \%$ injury prevalence [26], compared to $56.6 \%$ in the present study. This lower injury prevalence in retired GB Olympians may in part reflect a higher injury prevalence among Winter Olympians in the ROMHS cohort and a reduced risk of exposure to injury in $G B$ Olympians due to fewer years of participating within Olympic sports (mean $9.8 \pm 6.2$ years), compared to the ROMHS (mean $10.4 \pm 5.6$ years). However, the number of injuries reported per Olympian was the same (1.1 injuries) after excluding non-sports injuries with a higher injury prevalence in training.

The results of this study expand previous observations of injury data in current athletes. The most prominent injury locations in retired GB Olympians were the knee, lumbar spine, and shoulder. These findings accord with data from retired athletes [26], and current athletes at the 2010 Winter Olympic Games in Vancouver [4]. In comparison, the thigh, knee, and lumbar spine were the most prominent injury locations at the Sochi 2014 Winter Olympic Games [27], compared to the knee, thigh, and ankle at the Rio de Janeiro 2016 Olympic Summer Games [9]. In our study population, injury was more prevalent at the knee and lumbar spine in both summer and winter sports, particularly among those competing in weight-bearing loading sports. Injury prevention initiatives should consider that sport injuries at the hip, knee, and lumbar spine were associated with a higher prevalence of morbidity in later life compared to those without injury.

The most frequent injury types were ligament sprain, traumatic fractures, and muscle injury with no differences between sexes in the type of injury except for fractures. Females self-reported a lower Olympic career BMI and approximately twice as many stress fractures compared to males. This may be a result of impaired physiological functioning caused by low energy availability (LEA) which underpins relative energy deficiency syndrome (RED-S). This syndrome can lead to impaired physiological functioning and impairments in bone health, immunity, menstrual function, protein synthesis, and cardiovascular health [28]. Prior studies have shown that specific athletic populations at an increased risk for lower bone mineral density include swimmers, runners, 





and cyclists [29-32]. Our study confirms a history of stress fractures among female swimmers (lumbar spine), runners (ankle and tibia), and extends this to rowers (ribs) and gymnasts (tibia). It should be noted that our study does not show causation with LEA and warrants further investigation to determine and to mitigate the risk of stress fractures in female athletic populations.

Our study illustrates that the prevalence of fractures, dislocations, severe head, and cervical spine injuries were greater among retired athletes from winter sports, particularly those with high-speed disciplines such as skiing, skating, and snowboarding. This trend is supported by data in the literature that indicates severe injuries are higher at the Winter Olympics. The number of head injuries (sport-related concussion) in 2018 PyeongChang (1.72 per 1000 athletes) and 2010 Vancouver (7.8 per 1000 participating athletes) were higher compared to 2008 Beijing (1.09 per 1000 registered athletes), 2012 London (0.57 per 1000 athletes), and 2016 Rio de Janeiro (1.06 per 1000 athletes) [3, 4, 8-10]. In total, we confirmed 10 neck injuries, 16 to the head/face, and 2 severe sport-related concussion injuries (3.08 per 1000 athletes).

Injury prevalence was highest in field athletics, gymnastics, track athletics, and lowest for canoeing, swimming, and sailing (sports with $\mathrm{n} \geqq 15$ participants). During Rio de Janeiro 2016 Olympic Games, the percentage of injured athletes was also high in athletics and artistic gymnastics, and low in canoeing (slalom and sprint), sailing, and swimming [9]. In the 2012 London Olympic Games, injury prevalence was low for canoeing (slalom and sprint) and swimming, but high for athletics, synchronised swimming, water polo, and sailing [10]. The percentage of athletes injured was highest in skiing and snowboarding disciplines at the 2014 and 2018 Winter Olympic Games [8, 21].

The present study illustrates that approximately one in two retired GB Olympians reported current musculoskeletal pain, irrespective of the underlying structural changes associated with OA. Knee pain prevalence (23.4\%) was lower compared to $27.3 \%$ in retired male international athletes [16], and $52.2 \%$ in ex-football players $[15,33]$. Osteoarthritis was most prevalent at the knee joint (12.5\%), the hip joint (10.2\%), and the lumbar spine (4.5\%). For the hip and knee, this is lower compared to $14.2 \%$ and $19.4 \%$ reported in 664 former male elite athletes [14], and $20.2 \%$ and $27.3 \%$ in 301 former athletes competing in power sports (boxers, wrestlers, weight lifters, throwers) [16]. The prevalence of OA was lower compared to those reported among former professional football players $-18.8 \%$ at the lumbar spine [34], $13.2 \%$ at the hip joint [35], and $21.3 \%$ at the knee [36].

In our study population, injury was associated with early onset of $\mathrm{OA}$ in the lower limbs (hip, knee, and ankle) before 45 years and OA-joint replacement at the hip and knee. The risk of PTOA at the knee before 45 years was reported to be high among team sport athletes (soccer, ice hockey, and basketball players) [16], and soccer players and weight-lifters [17]. Our study extends this knowledge to the lower limb joints and retired athletes competing in weight-bearing loading sports including athletics (running, jumping, throwing events), hockey, figure skating, and skiing.

There are several potential explanations for differences in the prevalence of pain and OA in the reviewed literature. There are different definitions and different diagnostic criteria for OA, including radiography $[15,33$, 37], arthroscopy [38], or a self-reported physiciandiagnosis OA [20,36]. There are different sports involved in the studies in the literature that may potentially affect the prevalence of OA. There are also variations in methodological procedures with some studies measuring the prevalence rate at each limb [36]. Whereas other studies measure the prevalence of OA according to the most severe limb [37].

This study examined the consequences of injury associated with retirement from Olympic sports. This study is not without limitations. Firstly, there is a possibility that retired GB Olympians who had previously sustained an injury would have a greater propensity to partake in this study. To mitigate the risk of recruitment bias, we invited all GB Olympians on the BOA Olympian database the opportunity to complete and return a questionnaire. We made strenuous efforts to mail the questionnaire to retired athletes living in 30 different countries. Secondly, the cross-sectional design may be limited by recall bias, particularly as the majority of participants had retired over 5 years ago. We attempted to offset this risk by including an injury definition that asked participants to recall only significant injuries they had sustained and were more likely to recall. Thirdly, the medical attention injury definition used in surveillance studies at the current Olympic Games may allow sports with a high frequency of minor injuries to present with a higher injury prevalence. Finally, the athlete numbers in several sports were too low to provide a reasonable comparison. Further investigation in these sports with athletes from other National Olympic Committees is recommended.

\section{Conclusion}

The lifetime prevalence of Olympic career injury was $56.6 \%$, with $19.5 \%$ of participants retiring early from Olympic sport due to injury. Sport injury was the main attributable factor associated with self-reported current pain and/or OA at the hip, knee, and lumbar spine, early PTOA in the lower limb (hip, knee, ankle), and joint replacement at the hip and knee. Prevention strategies to 
reduce morbidity and improve long-term health should aim to reduce injuries specifically at the hip, knee, and lumbar spine. Further study is recommended to determine if Olympic athletes are at greater risk of pain, OA, and joint replacement compared with the general population.

\section{Abbreviations}

GB: Great Britain; OA: Osteoarthritis; IOC: International Olympic Committee; BOA: British Olympic Association; OR: Odds ratio; aOR: Adjusted odds ratio; Cl: Confidence interval; ROMHS: Retired Olympian Musculoskeletal Health Study; RED-S: Relative energy deficiency syndrome; LEA: Low energy availability

\section{Acknowledgements}

We would like to thank the staff at the Centre for Sport, Exercise and Osteoarthritis Research Versus Arthritis (formerly the Arthritis Research UK Centre for Sport, Exercise and Osteoarthritis), the University of Nottingham, the British Olympic Association (BOA) Athletes' Commission, and all the participants who contributed to this study. We would like to thank Sarah Winckless and Christine Bower for their invaluable assistance with distributing the survey. This work was supported by Versus Arthritis [grant number 20194], funding gratefully received from the Centre for Sport, Exercise and Osteoarthritis Research Versus Arthritis.

\section{Authors' contributions}

DJC conceived and contributed to the design of the study, distributed the survey, collected, analysed and interpreted the data, and drafted the manuscript. MEB assisted with the conception of the study, with accessing the study participants, with the distribution of the survey, and with the interpretation of the data. MEB critically revised the manuscript and gave final approval of the version to be published. $\mathrm{MO}$ assisted with the analysis and interpretation of the data. MO critically revised the manuscript and gave final approval of the version to be published. DP assisted with the conception of the study, with the distribution of the survey, and with the interpretation of the data. DP critically revised the manuscript and gave final approval of the version to be published. All authors read and approved the final manuscript.

\section{Funding}

This work was supported by Versus Arthritis [grant number 20194], and funding was gratefully received from the Centre for Sport, Exercise and Osteoarthritis Research Versus Arthritis.

\section{Availability of Data and Materials}

An anonymised summary of the dataset generated and analysed during the current study may be available from the corresponding author on reasonable request.

\section{Declarations}

\section{Ethics Approval and Consent to Participate}

This study was approved by the University of Nottingham Research Ethics Committee (Reference No: K13022014). All procedures involving research participants were in accordance with the ethical standards of the university institution review board and with the 1964 Helsinki Declaration and its later amendments. It was made explicitly clear to participants that by completing and returning a questionnaire, they gave implied consent for their data to be used anonymously for the purposes of this study.

\section{Consent for Publication}

It was made explicitly clear to participants that by completing and returning a questionnaire, they gave implied consent for publication from the dataset.

\section{Competing Interests}

The authors, Dale Cooper, Mark Batt, Mary O'Hanlon, and Debbie Palmer, declare that they have no competing interests.

\section{Author details}

${ }^{1}$ School of Allied Health Professions, Keele University, Keele, Staffordshire, UK. ${ }^{2}$ Centre for Sport, Exercise and Osteoarthritis Research Versus Arthritis, Nottingham University Hospitals NHS Trust, Nottingham, UK. ${ }^{3}$ Academic Department of Orthopaedics, Trauma and Sports Medicine, University of Nottingham, Nottingham, UK. ${ }^{4}$ Institute for Sport, PE and Health Sciences Moray House School of Education and Sport, University of Edinburgh, Edinburgh, UK.

Received: 11 March 2021 Accepted: 20 June 2021

Published online: 31 July 2021

\section{References}

1. Engebretsen L, Bahr R, Cook JL, Derman W, Emery CA, Finch CF, et al. The IOC centres of excellence bring prevention to sports medicine. Br J Sports Med. 2014;48(17):1270-5. https://doi.org/10.1136/bjsports-2014-093992.

2. Ljungqvist A. Sports injury prevention: a key mandate for the $I O C$. Br J Sports Med. 2008;42(6):391

3. Junge A, Engebretsen L, Mountjoy ML, Alonso JM, Renström PAF, Aubry MJ, et al. Sports injuries during the Summer Olympic Games 2008. Am J Sports Med. 2009;37(11):2165-72. https://doi.org/10.1177/0363546509339357.

4. Engebretsen L, Steffen K, Alonso JM, Aubry M, Dvorak J, Junge A, et al. Sports injuries and illnesses during the Winter Olympic Games 2010. Br J Sports Med. 2010;44(11):772-80. https://doi.org/10.1136/bjsm.2010.076992.

5. Junge A, Dvořák J. Football injuries during the 2014 FIFA World Cup. Br J Sports Med. 2015;49(9):599-602. https://doi.org/10.1136/bjsports-2014-0944 69.

6. Ekstrand J, Hagglund $M$, Walden $M$. Injury incidence and injury patterns in professional football: the UEFA injury study. Br J Sports Med. 2011;45(7):5538. https://doi.org/10.1136/bjsm.2009.060582.

7. Loosemore M, Lightfoot J, Palmer-Green D, Gatt I, Blizon J, Beardsley C. Boxing injury epidemiology in the Great Britain team: a 5-year surveillance study of medically diagnosed injury incidence and outcome. Br J Sports Med. 2015;49(17):1100-7. https://doi.org/10.1136/bjsports-2015-094755.

8. Soligard T, Palmer D, Steffen K, Lopes AD, Grant ME, Kim D, et al. Sports injury and illness incidence in the PyeongChang 2018 Olympic Winter Games: a prospective study of 2914 athletes from 92 countries. Br J Sports Med. 2019;53(17):1085-92. https://doi.org/10.1136/bjsports-2018-100236.

9. Soligard T, Steffen K, Palmer D, Alonso JM, Bahr R, Lopes AD, et al. Sports injury and illness incidence in the Rio de Janeiro 2016 Olympic Summer Games: a prospective study of 11274 athletes from 207 countries. $\mathrm{Br} J$ Sports Med. 2017;51(17):1265-71. https://doi.org/10.1136/bjsports-2017097956.

10. Engebretsen L, Soligard T, Steffen K, Alonso JM, Aubry M, Budgett R, et al. Sports injuries and illnesses during the London Summer Olympic Games 2012. Br J Sports Med. 2013;47(7):407-14. https://doi.org/10.1136/bjsports-2 013-092380

11. Kettunen JA, Kujala UM, Kaprio J, Backmand H, Peltonen M, Eriksson JG, et al. All-cause and disease-specific mortality among male, former elite athletes: an average 50-year follow-up. British J Sport Med. 2015;49(13):8937. https://doi.org/10.1136/bjsports-2013-093347.

12. Jones ME, Davies MAM, Leyland KM, Delmestri A, Porter A, Ratcliffe J, et al. Osteoarthritis and other long-term health conditions in former elite cricketers. J Sci Med Sport. 2018;21(6):558-63. https://doi.org/10.1016/j.jsa ms.2017.10.013.

13. Davies MAM, Judge DA, Delmestri A, Kemp S, Stokes KA, Arden NK, et al. Health amongst former rugby union players: a cross-sectional study of morbidity and health-related quality of life. Sci Rep. 2017;7(1):1-11.

14. Tveit M, Rosengren BE, Nilsson J, Karlsson MK. Former male elite athletes have a higher prevalence of osteoarthritis and arthroplasty in the hip and knee than expected. Am J Sports Med. 2012;40(3):527-33. https://doi.org/1 $0.1177 / 0363546511429278$.

15. Fernandes GS, Parekh SM, Moses J, Fuller C, Scammell B, Batt ME, et al. Prevalence of knee pain, radiographic osteoarthritis and arthroplasty in retired professional footballers compared with men in the general population: a cross-sectional study. Br J Sports Med. 2018;52(10):678-83. https://doi.org/10.1136/bjsports-2017-097503.

16. Kettunen JA, Kujala UM, Kaprio J, Koskenvuo M, Sarna S. Lower-limb function among former elite male athletes. Am J Sports Med. 2001;29(1):28. https://doi.org/10.1177/03635465010290010801. 
17. Kujala UM, Kettunen J, Paananen H, Aalto T, Battie MC, Impivaara O, et al. Knee osteoarthritis in former runners, soccer players, weight lifters, and shooters. Arthritis Rheum. 1995;38(4):539-46. https://doi.org/10.1002/art.1 780380413.

18. Whittaker JL, Roos EM. A pragmatic approach to prevent post-traumatic osteoarthritis after sport or exercise-related joint injury. Best Pract Res Clin Rheumatol. 2019;33(1):158-71. https://doi.org/10.1016/j.berh.2019.02.008.

19. Kujala UM, Kaprio J, Sarna S. Osteoarthritis of weight bearing joints of lower limbs in former élite male athletes. BMJ. 1994;308(6923):231-4. https://doi. org/10.1136/bmj.308.6923.231.

20. Cooper DJ, Scammell BE, Batt ME, Palmer D. Factors associated with pain and osteoarthritis at the hip and knee in Great Britain's Olympians: a crosssectional study. Br J Sports Med. 2018;52(17):1101-8. https://doi.org/10.1136/ bjsports-2017-098315.

21. Soligard T, Steffen K, Palmer-Green D, Aubry M, Grant ME, Meeuwisse W, et al. Sports injuries and illnesses in the Sochi 2014 Olympic Winter Games. Br J Sports Med. 2015;49(7):441-7. https://doi.org/10.1136/bjsports-2014-094 538.

22. Junge A, Engebretsen L, Alonso JM, Renström P, Mountjoy M, Aubry M, et al. Injury surveillance in multi-sport events: the International Olympic Committee approach. Br J Sports Med. 2008;42(6):413-21. https://doi.org/1 $0.1136 /$ bjsm.2008.046631

23. Hardt J, Jacobsen C, Goldberg J, Nickel R, Buchwald D. Prevalence of chronic pain in a representative sample in the United States. Pain Med. 2008;9(7):803-12. https://doi.org/10.1111/j.1526-4637.2008.00425.x.

24. Lacey RJ, Lewis M, Jordan K, Jinks C, Sim J. Interrater reliability of scoring of pain drawings in a self-report health survey. Spine (Phila Pa 1976). 2005; 30(16):E455-8. https://doi.org/10.1097/01.brs.0000174274.38485.ee.

25. Fuller CW, Bahr R, Dick RW, Meeuwisse WH. A framework for recording recurrences, reinjuries, and exacerbations in injury surveillance. Clin J Sport Med. 2007;17(3):197-200. https://doi.org/10.1097/JSM.0b013e3180471b89.

26. Palmer D, Cooper D, Emery C, Batt M, Engebretsen L, Scammell B, et al. Selfreported sports injuries and later-life health status in 3357 retired Olympians from 131 countries: a cross-sectional survey among those competing in the games between London 1948 and PyeongChang 2018. Br J Sports Med. 2021:55(1):46-53. https://doi.org/10.1136/bjsports-2019-101772.

27. Palmer-Green D, Elliott N. Sports injury and illness epidemiology: Great Britain Olympic Team (TeamGB) surveillance during the Sochi 2014 Winter Olympic Games. Br J Sports Med. 2015;49(1):25-9. https://doi.org/10.1136/ bjsports-2014-094206.

28. Mountjoy M, Sundgot-Borgen JK, Burke LM, Ackerman KE, Blauwet $C$, Constantini $\mathrm{N}$, et al. IOC consensus statement on relative energy deficiency in sport (RED-S): 2018 update. Br J Sports Med. 2018;52(11):687-97. https:// doi.org/10.1136/bjsports-2018-099193.

29. Tenforde AS, Fredericson M, Sayres LC, Cutti P, Sainani KL. Identifying sexspecific risk factors for low bone mineral density in adolescent runners. Am J Sports Med. 2015;43(6):1494-504. https://doi.org/10.1177/036354651 5572142.

30. Barrack MT, Rauh MJ, Nichols JF. Prevalence of and traits associated with low BMD among female adolescent runners. Med Sci Sports Exerc. 2008; 40(12):2015-21. https://doi.org/10.1249/MSS.0b013e3181822ea0.

31. Nichols JF, Rauh MJ. Longitudinal changes in bone mineral density in male Master cyclists and nonathletes. J Strength Cond Res. 2011;25(3):727-34. https://doi.org/10.1519/JSC.0b013e3181c6a116.

32. Morel J, Combe B, Francisco J, Bernard J. Bone mineral density of 704 amateur sportsmen involved in different physical activities. Osteoporos Int. 2001;12(2):152-7. https://doi.org/10.1007/s001980170148.

33. Parekh SM, Fernandes GS, Moses JP, Fuller CW, Scammell BE, Batt ME, et al. Risk factors for knee osteoarthritis in retired professional footballers. Clin J Sport Med. 2019;10:1097.

34. Turner AP, Barlow JH, Heathcote-Elliott C. Long-term health impact of playing professional football in the United Kingdom. Br J Sports Med. 2000; 34(5):332-6. https://doi.org/10.1136/bjsm.34.5.332.

35. Shepard GJ, Banks AJ, Ryan WG. Ex-professional association footballers have an increased prevalence of osteoarthritis of the hip compared with age matched controls despite not having sustained notable hip injuries. $\mathrm{Br}$ J Sports Med. 2003;37(1):80-1. https://doi.org/10.1136/bjsm.37.1.80.

36. Drawer $\mathrm{S}$, Fuller CW. Propensity for osteoarthritis and lower limb joint pain in retired professional soccer players. Br J Sports Med. 2001;35(6):402-8. https://doi.org/10.1136/bjsm.35.6.402.
37. Elleuch MH, Guermazi M, Mezghanni M, Ghroubi S, Fki H, Mefteh S, et al. Knee osteoarthritis in 50 former top-level footballers: a comparative (control group) study. Ann Readapt Med Phys. 2008;51(3):174-8. https://doi.org/10.1 016/j.annrmp.2008.01.003.

38. Nebelung W, Wuschech $\mathrm{H}$. Thirty-five years of follow-up of anterior cruciate ligament-deficient knees in high-level athletes. Arthrosc J Arthrosc Relat Surg. 2005;21(6):696-702. https://doi.org/10.1016/j.arthro.2005.03.010.

\section{Publisher's Note}

Springer Nature remains neutral with regard to jurisdictional claims in published maps and institutional affiliations.

\section{Submit your manuscript to a SpringerOpen ${ }^{\circ}$ journal and benefit from:}

- Convenient online submission

- Rigorous peer review

- Open access: articles freely available online

- High visibility within the field

- Retaining the copyright to your article

Submit your next manuscript at $\boldsymbol{\nabla}$ springeropen.com 